\title{
PRODUÇÃO DE MUDAS DE ESPÉCIES ARBÓREAS NATIVAS COM COMBINAÇÕES DE ADUBOS DE LIBERAÇÃO CONTROLADA E PRONTAMENTE SOLÚVEIS ${ }^{1}$
}

Sebastião Pires de Moraes Neto², José Leonardo de Moraes Gonçalves³, Carlos José Rodrigues ${ }^{4}$, Washington Luiz de Azevedo Geres ${ }^{4}$, Fabiane Ducatti ${ }^{5}$ e José Hamilton de Aguirre Jr. ${ }^{6}$

\begin{abstract}
RESUMO - O objetivo deste estudo foi testar diversas doses e fontes de adubo para verificar o crescimento de mudas de cinco espécies arbóreas nativas. As espécies utilizadas foram as pioneiras Guazuma ulmifolia (mutambo) e Croton floribundus (capixingui), as secundárias iniciais Peltophorum dubium (canafístula) e Gallesia integrifolia (pau-d'alho) e a clímax Myroxylon peruiferum (cabreúva). Os tratamentos consistiram principalmente na utilização de adubo de liberação controlada (ALC, 19-06-10, $\mathrm{N}_{-} \mathrm{P}_{2} \mathrm{O}_{5}-\mathrm{K}_{2} \mathrm{O}$ ) de maneira pura ou em mistura com adubo em pó (AP, 14-16-18), ou AP mais adubação de cobertura periódica (ACP, 19-06-20), comparados com adubação convencional (adubação de base mais adubação de cobertura com sulfato de amônio e cloreto de potássio) e testemunha (sem adubação). O substrato utilizado foi composto por $60 \%$ de húmus de minhoca, $30 \%$ de casca de arroz carbonizada e $10 \%$ de terra de subsolo, em base volumétrica. O recipiente que comportou o substrato foi o tubete de polipropileno de $50 \mathrm{~cm}^{3}$ de capacidade. Entre os tratamentos que produziram mudas de boa qualidade ao final do experimento, para cada espécie, aqueles com doses de 3,2 e 4,8 $\mathrm{kg}$ de adubo de liberação controlada por metro cúbico de substrato foram comuns a todas as espécies e, adicionalmente, resultaram em dimensões apropriadas para plantio no campo em menor tempo do que os outros tratamentos.
\end{abstract}

Palavras-chave: Viveiro, nutrição e espécies arbóreas da Floresta Atlântica.

\section{PRODUCTION OF SEEDLINGS OF NATIVE TREE SPECIES COMBINED WITH CONTROLLED-RELEASE AND SOLUBLE READY FERTILIZERS}

\begin{abstract}
This study aimed to test different doses and sources of fertilizers to verify the growth of seedlings of five native tree species. The species used were the pioneers Guazuma ulmifolia (mutambo) and Croton floribundus (capixingui), the early secondaries Peltophorum dubium (canafístula) and Gallesia integrifolia (pau-d'alho) and the climax Myroxylon peruiferum (cabreúva). The treatments consisted mainly of the use of controlledrelease fertilizers (CRF, 19-06-10, $\left.\mathrm{N}-\mathrm{P}_{2} \mathrm{O}_{5}-\mathrm{K}_{2} \mathrm{O}\right)$ alone or combined with powder fertilizers (PF, 14-16-18) or with PF and periodic covering fertilization (PCF, 19-06-20) compared with conventional fertilization (base fertilization plus covering fertilization with ammnonium sulfate and potassium chloride) and control (without fertilization). The substrate was composed of $60 \%$ earthworm humus $+30 \%$ carbonized rice husk and $10 \%$ subsoil, by volume. Tubes of $50 \mathrm{~cm}^{3}$ capacity were used as containers. Among the treatments that produced seedlings of good quality at the end of the experiment for each species, the treatments with doses of 3,2 and $4,8 \mathrm{~kg}$ of controlled-release fertilizers by $\mathrm{m}^{3}$ of substrate were common to all species, also resulting in appropriate dimensions for field planting at a shorter time than the other treatments.
\end{abstract}

Key words: $\quad$ Nursery, nutrition, and Atlantic Forest tree species.

1 Recebido para publicação em 7.8.2002.

Aceito para publicação em 4.11.2003.

Convênio entre CESP/IPEF/ESALQ

2 Dr. em Ciências Biológicas, < spmneto@bol.com.br>. ${ }^{3}$ Prof. Dr. do Dep. de Ciências Florestais da Escola Superior de Agricultura "Luiz de Queiroz" - ESALQ-USP, <jlmgonca @ carpa.ciagri.usp.br>. ${ }^{4}$ Engenheiro da CESP. ${ }^{5}$ M.S. em Engenharia Florestal - ESALQ-USP; ${ }^{6}$ Engenheiro Florestal - ESALQ-USP. 


\section{INTRODUÇÃO}

A fertilização do substrato é uma das fases mais importantes em um programa de produção de mudas de espécies arbóreas. Existem no mercado inúmeros tipos de adubos, que variam na sua composição, forma (pó, grânulos e encapsulados) e solubilidade. Dentre esses adubos, os de liberação controlada (adubos encapsulados) estão começando a ser utilizados nos viveiros do Brasil. Apesar de seu custo elevado, têm sido economicamente viáveis para uso em viveiros florestais (Rodella \& Alcarde, 2000), pois, considerando-se que $1 \mathrm{~m}^{3}$ de substrato preenche cerca de 16.000 tubetes de polipropileno de $50 \mathrm{~cm}^{3}$ e que seriam gastos em torno de $5 \mathrm{~kg}$ de adubo por metro cúbico de substrato, o custo unitário por muda seria mínimo, evitando, portanto, investimentos em sistemas de fertirrigação e diminuindo a mão-de-obra, caso a adubação seja feita manualmente com regadores.

Contudo, é necessário conhecer o comportamento das espécies quanto à rapidez de crescimento, porque, em geral, as espécies da categoria sucessional das pioneiras (Budowski, 1965) têm um ciclo de produção mais curto que o das espécies de sucessão mais tardia, e se for aplicado um adubo de liberação controlada (ALC) de tempo de duração de 3-4 meses ele pode ter pouco efeito sobre as últimas. Por outro lado, a lixiviação de nitrogênio em ALC pode ser superior a aplicações freqüentes e pequenas de fertilizantes solúveis em água (Cox, 1993).

Os ALC, em geral, produzem maior crescimento das plantas e menos lixiviação que os mesmos fertilizantes não-encapsulados (Cole \& Dole, 1997). A perda de nutrientes lixiviados para os ALC aumenta com o maior volume de lixiviável (Huett \& Morris, 1999) e a menor capacidade de retenção de água e CTC do substrato (Hamilton \& Johnson, 1978).

O objetivo deste trabalho foi analisar o crescimento de mudas de cinco espécies arbóreas nativas, utilizando adubos de liberação controlada de nutrientes e prontamente solúveis.

\section{MATERIAL E MÉTODOS}

O experimento foi instalado no viveiro florestal da Usina Hidrelétrica de Jupiá, em Três Lagoas-MS. As temperaturas e umidades relativas médias do ar das máximas e das mínimas, no período experimental, encontram-se no Quadro 1. As cinco espécies utilizadas neste estudo, pertencentes a diferentes categorias sucessionais, foram selecionadas por serem usadas nos plantios realizados no entorno dos reservatórios da CESP (Quadro 2).

Para cada uma das espécies foi instalado um ensaio, constituído por 11 tratamentos de adubação (Quadro 3), dispostos em blocos ao acaso, com três repetições, sendo cada parcela composta por 32 mudas.

As sementes de todas as espécies foram germinadas em canteiros e repicadas para tubetes de polipropileno com volume útil de $50 \mathrm{~cm}^{3}$ (12 cm de comprimento, $3 \mathrm{~cm}$ de diâmetro superior e $1 \mathrm{~cm}$ de diâmetro na parte inferior). O substrato consistiu de $60 \%$ de húmus de minhoca, $30 \%$ de casca de arroz carbonizada e $10 \%$ de terra de subsolo em base volumétrica. A irrigação foi realizada

Quadro 1 - Precipitação e média das temperaturas e umidades relativas do ar máximas e mínimas diárias mensais no viveiro florestal da U.H.E. de Jupiá . Os valores entre parênteses são os desvios-padrão

Table 1 - Monthly rainfall and monthly means of maximum and minimum daily temperatures and air relative humidities at the Jupiá U.H.E. forest nursery. The values within parenthesis are the standard deviations

\begin{tabular}{|c|c|c|c|c|c|}
\hline \multirow[t]{2}{*}{ Mês } & Precipitação & $\begin{array}{c}\text { Temperatura } \\
\text { Máxima }\end{array}$ & $\begin{array}{c}\text { Temperatura } \\
\text { Mínima }\end{array}$ & $\begin{array}{l}\text { Umidade } \\
\text { Máxima }\end{array}$ & $\begin{array}{l}\text { Umidade } \\
\text { Mínima }\end{array}$ \\
\hline & $(\mathrm{mm})$ & \multicolumn{2}{|c|}{$\left({ }^{\circ} \mathrm{C}\right)$} & \multicolumn{2}{|c|}{$(\%)$} \\
\hline Dez. & 116 & $29,7(2,7)$ & $20,7(1,5)$ & $92,7(5,0)$ & $53,0(12,9)$ \\
\hline Jan. & 96 & $30,1(2,1)$ & $21,9(1,3)$ & $91,5(3,0)$ & $55,4(7,9)$ \\
\hline Fev. & 209 & $28,8(2,3)$ & $21,6(1,1)$ & $94,8(3,7)$ & $61,7(8,5)$ \\
\hline Mar. & 224 & $27,5(1,8)$ & $20,8(1,5)$ & $94,2(3,5)$ & $63,9(8,2)$ \\
\hline Abr. & 34 & $28,2(2,9)$ & $17,4(3,1)$ & $91,5(4,6)$ & $51,2(8,7)$ \\
\hline Maio & 32 & $25,5(2,9)$ & $14,3(3,4)$ & $95,3(2,8)$ & $51,6(8,9)$ \\
\hline Jun. & 4 & $25,8(3,1)$ & $14,4(2,8)$ & $94,3(5,0)$ & $51,7(9,3)$ \\
\hline Jul. & 45 & $23,1(4,7)$ & $10,8(5,7)$ & $93,8(5,6)$ & $48,6(10,2)$ \\
\hline
\end{tabular}


Quadro 2 - Características das espécies usadas

Table 2 - Characteristics of the species used

\begin{tabular}{|c|c|c|c|c|c|}
\hline \multirow[t]{2}{*}{ Espécie } & \multirow[t]{2}{*}{ Família/(Nome Popular) } & \multirow{2}{*}{$\begin{array}{c}\text { Categoria } \\
\text { Sucessional }\end{array}$} & $\begin{array}{l}\text { Altura } \\
\text { Adulta } \\
\end{array}$ & $\begin{array}{l}\text { Peso da } \\
\text { Semente }\end{array}$ & \multirow{2}{*}{$\begin{array}{l}\text { Dispersão da } \\
\text { Semente }\end{array}$} \\
\hline & & & $(\mathrm{m})$ & (mg) & \\
\hline Guazuma ulmifolia Lam. & $\begin{array}{l}\text { Sterculiaceae } \\
\text { (mutambo) }\end{array}$ & pioneira & $8-16$ & 6 & zoocórica \\
\hline Croton floribundus Spreng. & $\begin{array}{l}\text { Euphorbiaceae } \\
\text { (capixingui) }\end{array}$ & pioneira & $5-10$ & 36 & autocórica \\
\hline $\begin{array}{l}\text { Peltophorum dubium } \\
\text { (Spreng.) Taub. }\end{array}$ & $\begin{array}{l}\text { Leguminosae } \\
\text { Caesalpinioideae } \\
\text { (canafístula) }\end{array}$ & sec. inicial & $15-25$ & 50 & anemocórica \\
\hline $\begin{array}{l}\text { Gallesia integrifolia } \\
\text { (Spreng.) Harms }\end{array}$ & $\begin{array}{l}\text { Phytolaccaceae } \\
\text { (pau-d'alho) }\end{array}$ & sec. Inicial & $15-30$ & 83 & anemocórica \\
\hline Myroxylon peruiferum L. f. & $\begin{array}{l}\text { Leguminosae Papilionoideae } \\
\text { (cabreúva) }\end{array}$ & clímax & $10-20$ & 580 & anemocórica \\
\hline
\end{tabular}

Quadro 3 - Tratamentos de adubação utilizados para cada uma das cinco espécies Table 3 - Fertilization treatments used for each one of the five species

\begin{tabular}{|c|c|c|c|c|c|c|c|c|c|}
\hline \multirow{2}{*}{ Trat. } & \multicolumn{3}{|c|}{$\mathrm{ALC}^{1 /} 19-06-10\left(\mathrm{~kg} / \mathrm{m}^{3}\right)$} & \multicolumn{3}{|c|}{$\mathrm{AP}^{2 /} 14-16-18\left(\mathrm{~kg} / \mathrm{m}^{3}\right)$} & \multicolumn{3}{|c|}{$\mathrm{ACP}^{3 /} 19-06-20(\mathrm{~g} / \mathrm{l})$} \\
\hline & 1,6 & 3,2 & 4,8 & 0,75 & 1,5 & 2,25 & 0,5 & 1,0 & 2,0 \\
\hline $1^{\frac{4}{\prime}}$ & - & - & - & - & - & - & - & - & - \\
\hline 2 & $\mathrm{x}$ & - & - & - & - & - & - & - & - \\
\hline 3 & - & $\mathrm{x}$ & - & - & - & - & - & - & - \\
\hline 4 & - & - & $\mathrm{x}$ & - & - & - & - & - & - \\
\hline $5^{-5 /}$ & - & - & - & - & - & - & - & - & - \\
\hline 6 & $\mathrm{x}$ & - & - & $\mathrm{x}$ & - & - & - & - & - \\
\hline 7 & $\mathrm{x}$ & - & - & - & $\mathrm{x}$ & - & - & - & - \\
\hline 8 & $\mathrm{x}$ & - & - & - & - & $\mathrm{x}$ & - & - & - \\
\hline 9 & $\mathrm{x}$ & - & - & - & $\mathrm{x}$ & - & $\mathrm{x}$ & - & - \\
\hline 10 & $\mathrm{x}$ & - & - & - & $\mathrm{x}$ & - & - & $\mathrm{x}$ & - \\
\hline 11 & $\mathrm{X}$ & - & - & - & $\mathrm{X}$ & - & - & - & $\mathrm{X}$ \\
\hline
\end{tabular}

${ }^{1 /}$ Adubo de liberação controlada; ${ }^{1 /}$ adubo em pó; ${ }^{3 /}$ adubação de cobertura periódica - Foram feitas aplicações semanais (intervalos de sete dias) após 50 dias da repicagem. Foram usados 5 litros de solução para cada $1 \mathrm{~m}^{2}$ de canteiro; ${ }^{4 /}$ testemunha (sem adubação); ${ }^{5 /}$ adubação convencional no viveiro florestal da ESALQ: a) adubação de base: mistura de $750 \mathrm{~g}$ de sulfato de amônio mais $1,5 \mathrm{~kg}$ de superfosfato simples mais $170 \mathrm{~g}$ de cloreto de potássio e $150 \mathrm{~g}$ de FTE BR $12 \mathrm{em} 1 \mathrm{~m}^{3}$ de substrato; b) adubação de cobertura: $10 \mathrm{~g}$ de sulfato de amônio (S.A.) e $3 \mathrm{~g}$ de cloreto de potássio (CP) por litro de água. As adubações de cobertura foram repetidas a cada 14 dias de intervalo, iniciado 15 dias após a repicagem. A adubação com S.A. foi feita a cada 14 dias e a com CP a cada 28 dias.

por microaspersão, três vezes ao dia, sendo cada aplicação de 15 minutos.

Os adubos de base usados foram o de liberação controlada (19-06-10), o em pó (14-16-18) e adubação convencional (descrita no rodapé do Quadro 3), e os adubos de cobertura foram o 19-06-20, usado em três tratamentos (T9, T10 e T11), o sulfato de amônio e o cloreto de potássio, usados somente em um tratamento (T5).

O adubo de liberação controlada (19-06-10) é revestido com uma resina biodegradável capaz de liberar 17\% de $\mathrm{N}, 5,4 \%$ de $\mathrm{P}_{2} \mathrm{O}_{5}$ e $9 \%$ de $\mathrm{K}_{2} \mathrm{O}$. O N total é constituído

R. Árvore, Viçosa-MG, v.27, n.6, p.779-789, 2003 
de $10,1 \%$ de $\mathrm{N}$-amônico e $8,9 \%$ de $\mathrm{N}$-nitrato. A formulação do adubo é derivada do nitrato de amônio, fosfato de amônio, fosfato de cálcio e sulfato de potássio. $\mathrm{O}$ adubo em pó 14 - 16 - 18 é constituído de $14 \% \mathrm{~N}, 16 \%$ $\mathrm{P}_{2} \mathrm{O}_{5}, 18 \% \mathrm{~K}_{2} \mathrm{O}, 8 \% \mathrm{~S}, 0,4 \% \mathrm{Mg}, 0,03 \% \mathrm{~B}, 0,2 \% \mathrm{Mo}$, $0,16 \% \mathrm{Mn}$ e $0,12 \% \mathrm{Cu}$. O adubo de cobertura 19-06-20 é constituído por $19 \% \mathrm{~N}, 6 \% \mathrm{P}_{2} \mathrm{O}_{5}, 20 \% \mathrm{~K}_{2} \mathrm{O}, 3 \% \mathrm{MgO}$, $3 \%$ S, $0,025 \%$ B, $0,004 \%$ Mo, $0,01 \%$ CuEDTA, $0,07 \%$ FeEDTA, 0,04\% MnEDTA, 0,025\% ZnEDTA.

Para determinação das características químicas da solução dos substratos, imediatamente antes de preencher os tubetes, um volume de $150 \mathrm{ml}$ foi medido em proveta, transferido para vidros de conserva, os quais receberam água deionizada na proporção volumétrica de 1 parte de substrato: 1,5 de água. Em seguida, os frascos foram postos em um mesa agitadora por 1 hora, na posição horizontal. Após 24 horas de repouso em posição vertical, o sobrenadante foi filtrado e usado nas análises. Não foram coletadas amostras dos tratamentos 9, 10 e 11, porque a adubação de base destes tratamentos é semelhante à do tratamento 7 (Quadro 4).

O material vegetal de 15 mudas por tratamento e espécie foi dividido em parte aérea (folhas + caule) e raiz e, em seguida, colocado em estufa a $65^{\circ} \mathrm{C}$, até atingir peso constante. Após a pesagem do material seco, foram escolhidos os sete tratamentos que representassem cada tipo de adubação utilizada e, dentro de algumas destas, as doses que propiciaram maior crescimento das mudas, para análise química da parte aérea das plantas, que foi previamente moída. Em seguida, determinou-se a concentração dos macronutrientes através de digestão nitroperclórica. A determinação de $\mathrm{N}$ foi realizada pelo método de Kjeldahl (Malavolta et al., 1997).

O experimento iniciou-se em 20 de dezembro de 1998 (para quatro espécies) e 8 de janeiro de 1999 para Gallesia integrifolia (pau-d'alho) e encerrou-se em 15 de julho de 1999. As espécies foram coletadas em idades distintas para determinação da biomassa seca, baseandose em experiência anterior dos tamanhos adequados que cada espécie atinge, em condições de tubete de $50 \mathrm{~cm}^{3}$, e em taxas decrescentes de crescimento das plantas, um pouco antes de sua estabilização, nos tratamentos onde foi aplicada adubação de cobertura. Assim, Guazuma ulmifolia e Croton floribundus foram coletadas aos 90 dias após a germinação, Peltophorum dubium aos 120 dias, Gallesia integrifolia aos 150 dias e Myroxylon peruiferum aos 180 dias. As medições do crescimento foram realizadas até os 180 dias após a germinação, em todas as espécies, para confirmação da estabilização do crescimento das mudas que foram coletadas antes deste prazo.

Foram utilizados os programas SAS (SAS INSTITUTE, 1990) para os cálculos estatísticos, ANOVA e diferença de médias. Quando necessário, foram feitas transformações dos dados para homogeneizar as variâncias e adequá-los à distribuição normal. A diferença entre as médias foi obtida pelo teste de Tukey, a 95\% de probabilidade (Steel \& Torrie, 1980).

Quadro 4 - Características químicas das soluções dos substratos (1 substrato: 1,5 água deionizada, v/v) nos diversos tratamentos, imediatamente antes da repicagem ${ }^{1 /}$

Table 4 - Chemical characteristics of substrate solutions (1 substrate:1,5 deionized water, $v / v$ ) in the different treatments, imediatelly before transplanting

\begin{tabular}{|c|c|c|c|c|c|c|c|c|c|}
\hline \multirow[t]{2}{*}{ Trat. } & \multirow{2}{*}{$\begin{array}{c}\mathrm{pH} \\
\mathrm{CaCl}_{2}{ }^{2 /}\end{array}$} & $\begin{array}{l}\text { Cond. } \\
\text { Elétrica }\end{array}$ & $\mathrm{N}-\mathrm{NH}_{4}$ & $\mathrm{~N}-\mathrm{NO}_{3}$ & $\mathrm{P}$ & $\mathrm{K}$ & $\mathrm{Ca}$ & $\mathrm{Mg}$ & $\mathrm{SO}_{4}$ \\
\hline & & $(\mathrm{mS} / \mathrm{cm})$ & \multicolumn{7}{|c|}{$(\mathrm{mg} / \mathrm{l})$} \\
\hline 1 & 5,8 & 1,65 & 0,3 & 2,1 & 20,3 & 179 & 197 & 57 & 143 \\
\hline 2 & 5,9 & 1,55 & 0,2 & 1,4 & 18,8 & 173 & 191 & 57 & 143 \\
\hline 3 & 5,8 & 1,50 & 0,4 & 1,7 & 17,7 & 164 & 170 & 57 & 127 \\
\hline 4 & 5,9 & 1,54 & 0,6 & 1,7 & 19,3 & 160 & 168 & 58 & 127 \\
\hline 5 & 5,5 & 2,35 & 21,6 & 1,6 & 50,0 & 198 & 256 & 59 & 473 \\
\hline 6 & 5,8 & 1,70 & 9,9 & 1,8 & 33,3 & 177 & 163 & 59 & 186 \\
\hline 7 & 5,5 & 1,90 & 21,7 & 1,7 & 47,9 & 199 & 146 & 57 & 279 \\
\hline 8 & 5,6 & 2,35 & 24,9 & 1,1 & 58,3 & 198 & 162 & 58 & 304 \\
\hline
\end{tabular}

${ }^{1 /}$ Análises químicas realizadas segundo os métodos da AMERICAN PUBLIC HEALTH ASSOCIATION (1976). ${ }^{2 /}$ 0,01 Mol/l. 


\section{RESULTADOS E DISCUSSÃO}

A Figura 1 mostra o crescimento em altura ao longo de 180 dias, para as cinco espécies, em alguns tratamentos representativos. As pioneiras Guazuma ulmifolia e Croton floribundus apresentaram o maior incremento em altura e praticamente estabilizaram seu crescimento aos 90 dias após a germinação. O Peltophorum dubium, nos tratamentos adubados, estabilizou o crescimento aos 120 dias, tendo os tratamentos 4,5 e 11 resultado em comportamento semelhante no crescimento em altura das mudas. A Gallesia integrifolia e o Myroxylon peruiferum estabilizaram o crescimento em altura aos 150 e 180 dias, respectivamente.

As mudas de Guazuma ulmifolia, Croton floribundus e Gallesia integrifolia apresentaram maior crescimento em altura nos tratamentos 3 e 4 (Quadro 5). O Peltophorum dubium apresentou os maiores valores em altura nos tratamentos $3,4,5,9,10$ e 11, que não diferiram entre si, enquanto o Myroxylon peruiferum mostrou maior crescimento nos tratamentos 4,5 e 11 .

Em relação ao crescimento em diâmetro das mudas (Quadro 5), percebe-se que os maiores valores para a Guazuma ulmifolia foram obtidos nos tratamentos 3 e 4, enquanto para o Croton floribundus e o Peltophorum dubium os tratamentos que mais se destacaram foram o 10 e o 11. A Gallesia integrifolia apresentou os maiores valores em diâmetro do coleto nos tratamentos 11, 3, 4, 5 e 9.

A biomassa seca da raiz (BSR) de Guazuma ulmifolia, Croton floribundus e Myroxylon peruiferum não difeririu entre os tratamentos (Quadro 5). A BSR de Peltophorum dubium e Gallesia integrifolia nos tratamentos 3,10 e 11 diferiram da BSR do tratamento 1 (testemunha).

A biomassa seca da parte aérea (BSPA) de Guazuma ulmifolia (Quadro 5) no tratamento 4 só não diferiu da BSPA dos tratamentos 3 e 5. A BSPA de Croton floribundus, nos tratamentos 3, 4, 7 e 9, só diferiu da testemunha. A BSPA de Peltophorum dubium no tratamento 11 não diferiu da dos tratamentos 3, 4, 5, 6 e 10. A BSPA de Gallesia integrifolia, nos tratamentos 3 , 4 e 11, diferiu da BSPA do tratamento 9. A BSPA de Myroxylon peruiferum, nos tratamentos 3, 4, 10 e 11, diferiu da do tratamento 8 .

A biomassa seca total (BST) de Guazuma ulmifolia (Quadro 5), nos tratamentos 3, 4 e 5, diferiu da BST do tratamento 9 e 1. A BST de Croton floribundus no tratamento 9 diferiu da BST da testemunha. A BST de Peltophorum dubium no tratamento 11 não diferiu da dos tratamentos 3, 4, 5, 7 e 10. A BST de Gallesia integrifolia no tratamento 4 não diferiu da dos tratamentos 3, 5, 9, 10 e 11. A BST de Myroxylon peruiferum no tratamento 11 não diferiu da dos tratamentos $2,3,4,5$, 6,9 e 10.

A razão entre raiz e parte aérea (R/P) de Guazuma ulmifolia e Myroxylon peruiferum (Quadro 5) na testemunha diferiu da dos demais tratamentos, com exceção dos tratamentos 2 e 6 para a primeira espécie. A razão R/P de Croton floribundus não diferiu entre os tratamentos. A razão R/P de Peltophorum dubium no tratamento 11 só diferiu da dos tratamentos 1 e 7 . A razão R/P de Gallesia integrifolia na testemunha não diferiu da dos tratamentos $2,6,7,9$ e 10 . A razão $\mathrm{R} / \mathrm{P}$ de Myroxylon peruiferum na testemunha diferiu da dos demais tratamentos.

A concentração de nutrientes na parte aérea das mudas das cinco espécies é apresentada no Quadro 6. As concentrações de $\mathrm{N}, \mathrm{K}$ e S na parte aérea das mudas no tratamento 5 foram maiores que no tratamento 7 , para todas as espécies, à exceção do S para a Guazuma ulmifolia, onde elas não diferiram. A concentraçao de $\mathrm{P}$ na parte aérea de Peltophorum dubium e Myroxylon peruiferum não apresentou diferença entre os tratamentos, enquanto para o Croton floribundus, a Guazuma ulmifolia e a Gallesia integrifolia houve a tendência de os tratamentos que utilizaram menos adubação apresentarem os maiores valores. As concentrações de $\mathrm{Ca}$ e $\mathrm{Mg}$ na parte aérea das mudas apresentaram tendência, para todas as espécies, de maiores valores nos tratamentos onde houve menos adubação.

No Quadro 7 está o conteúdo de nutrientes na parte aérea das mudas. Ao comparar os tratamentos 4 e 5 , constata-se que o conteúdo de N, Ca e Mg na parte aérea das mudas só diferiu para a Gallesia gorarema, enquanto para o K houve diferença para a Guazuma ulmifolia, a G. integrifolia e o Myroxylon peruiferum. Entre esses tratamentos, o conteúdo de $\mathrm{P}$ na parte aérea das mudas não diferiu para todas as espécies, e para o $\mathrm{S}$ só existiu diferença para o Croton floribundus e a G. integrifolia.

$\mathrm{O}$ crescimento em altura ao longo do experimento (Figura 1) mostra que a aplicação de adubo de liberação controlada (ALC) na dose de $4,8 \mathrm{~kg} / \mathrm{m}^{3}$ (tratamento 4) compatibilizou o desprendimento de nutrientes com a

R. Árvore, Viçosa-MG, v.27, n.6, p.779-789, 2003 

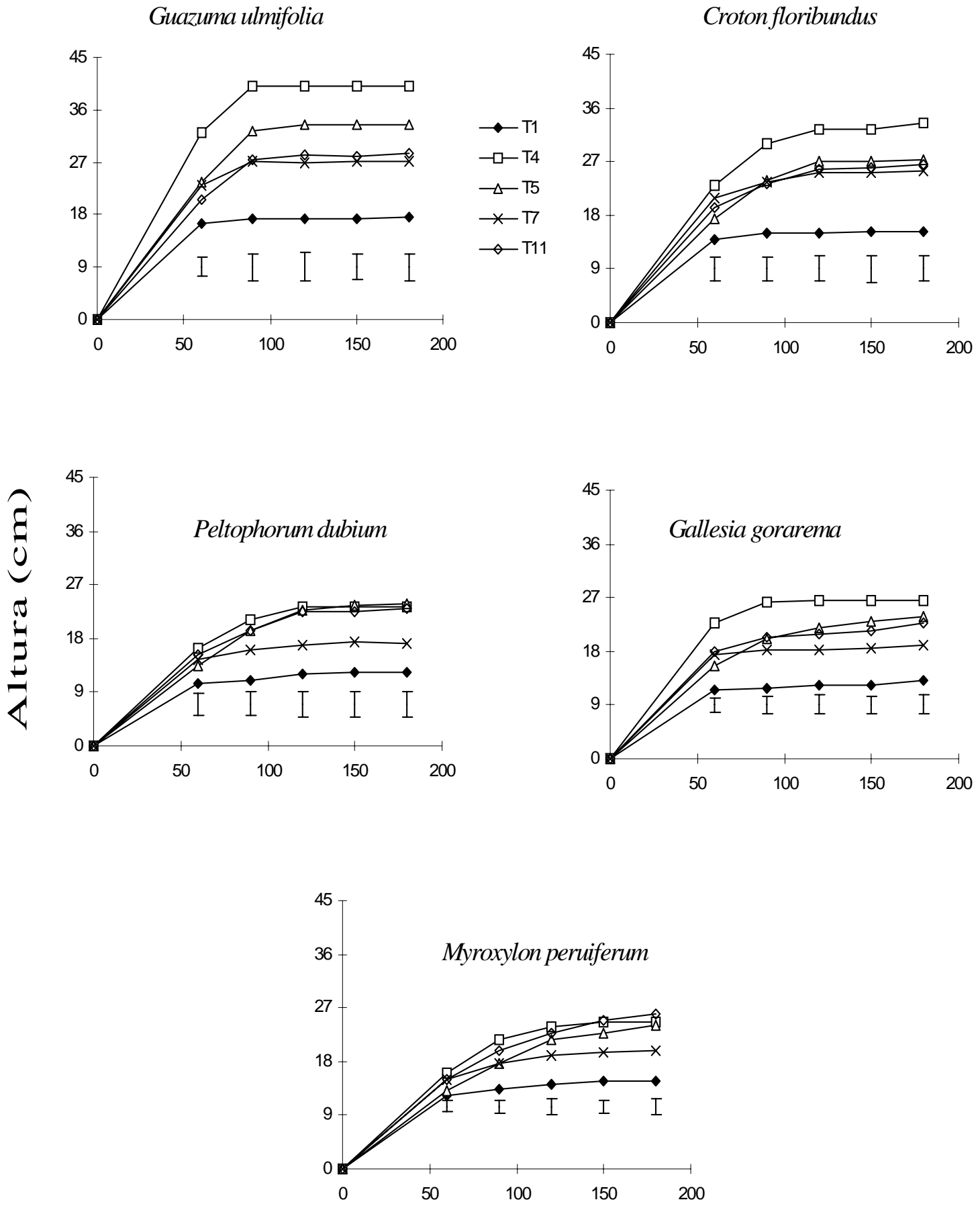

\section{Tempo (dias após a germinação)}

Figura 1 - Crescimento em altura das mudas das cinco espécies. As barras representam as diferenças mínimas significativas $(\mathrm{P} \leq 0,05)$ pelo teste de Tukey.

Figure 1 - Seedling height growth of the five species. The vertical bars represent the least significant differences $(P \leq 0.05)$, according to the Tukey test.

R. Árvore, Viçosa-MG, v.27, n.6, p.779-789, 2003 
Quadro 5 - Altura, diâmetro do coleto, biomassa seca da raiz (BSR), da parte aérea (BSPA) e total (BST) e razão entre raiz e parte aérea $(\mathrm{R} / \mathrm{P})$ das mudas das cinco espécies

Table 5 - Height, collar diameter, root dry biomass (BSR), shoot dry biomass (BSPA), total dry biomass (BST) and ratio between root and shoot $(R / P)$ of the five species seedlings

\begin{tabular}{|c|c|c|c|c|c|c|c|c|c|c|c|c|}
\hline \multirow{3}{*}{ Tr. } & ALT & DI & BSR & BSPA & BST & $\mathrm{R} / \mathrm{P}$ & ALT & DI & BSR & BSPA & BST & $\mathrm{R} / \mathrm{P}$ \\
\hline & $(\mathrm{cm})$ & $(\mathrm{mm})$ & \multicolumn{3}{|c|}{ (g) } & & $(\mathrm{cm})$ & $(\mathrm{mm})$ & \multicolumn{3}{|c|}{ (g) } & \\
\hline & \multicolumn{6}{|c|}{ Guazuma ulmifolia } & \multicolumn{6}{|c|}{ Croton floribundus } \\
\hline 1 & $18 \mathrm{f}$ & $3,1 \mathrm{~d}$ & $0,8 \mathrm{a}$ & $0,9 \mathrm{~d}$ & $1,7 \mathrm{c}$ & $0,92 \mathrm{a}$ & $15 \mathrm{~d}$ & $3,3 \mathrm{c}$ & $0,3 \mathrm{a}$ & $0,7 \mathrm{~b}$ & $1,0 \mathrm{~b}$ & $0,48 \mathrm{a}$ \\
\hline 2 & $28 \mathrm{de}$ & $4,0 \mathrm{bc}$ & $1,0 \mathrm{a}$ & $1,5 \mathrm{c}$ & $2,5 \mathrm{ac}$ & $0,69 \mathrm{ab}$ & $26 \mathrm{bc}$ & $4,1 \mathrm{~b}$ & $0,5 \mathrm{a}$ & $1,4 \mathrm{ab}$ & $1,9 \mathrm{ab}$ & $0,36 \mathrm{a}$ \\
\hline 3 & $37 \mathrm{ab}$ & $4,4 \mathrm{ab}$ & $1,1 \mathrm{a}$ & $2,2 \mathrm{ab}$ & $3,3 \mathrm{a}$ & $0,49 \mathrm{~b}$ & $30 \mathrm{ab}$ & $4,4 \mathrm{ab}$ & $0,6 \mathrm{a}$ & $1,7 \mathrm{a}$ & $2,3 \mathrm{ab}$ & $0,36 \mathrm{a}$ \\
\hline 4 & $40 \mathrm{a}$ & $4,8 \mathrm{a}$ & $1,1 \mathrm{a}$ & $2,3 \mathrm{a}$ & $3,4 \mathrm{a}$ & $0,46 \mathrm{~b}$ & $33 \mathrm{a}$ & $4,6 \mathrm{ab}$ & $0,5 \mathrm{a}$ & $1,7 \mathrm{a}$ & $2,2 \mathrm{ab}$ & $0,31 \mathrm{a}$ \\
\hline 5 & $33 \mathrm{bc}$ & $4,2 \mathrm{bc}$ & $1,2 \mathrm{a}$ & $2,2 a b$ & $3,4 \mathrm{a}$ & $0,54 \mathrm{~b}$ & $27 \mathrm{bc}$ & $4,3 \mathrm{ab}$ & $0,6 \mathrm{a}$ & $1,6 a b$ & $2,2 \mathrm{ab}$ & $0,38 \mathrm{a}$ \\
\hline 6 & $32 \mathrm{~cd}$ & $4,0 \mathrm{bc}$ & $1,2 \mathrm{a}$ & $1,6 \mathrm{c}$ & $2,8 \mathrm{ab}$ & $0,73 \mathrm{ab}$ & $25 \mathrm{c}$ & $4,1 \mathrm{~b}$ & $0,7 \mathrm{a}$ & $1,4 \mathrm{ab}$ & $2,1 \mathrm{ab}$ & $0,47 \mathrm{a}$ \\
\hline 7 & $27 \mathrm{de}$ & $3,9 \mathrm{c}$ & $0,7 \mathrm{a}$ & $1,2 \mathrm{~cd}$ & $2,0 \mathrm{bc}$ & $0,59 \mathrm{~b}$ & $25 \mathrm{c}$ & $4,1 \mathrm{~b}$ & $0,7 \mathrm{a}$ & $1,7 \mathrm{a}$ & $2,4 \mathrm{ab}$ & $0,42 \mathrm{a}$ \\
\hline 8 & $28 \mathrm{de}$ & $4,1 \mathrm{bc}$ & $0,9 \mathrm{a}$ & $1,4 \mathrm{c}$ & $2,4 \mathrm{ac}$ & $0,65 \mathrm{~b}$ & $25 \mathrm{c}$ & $4,2 \mathrm{~b}$ & $0,5 \mathrm{a}$ & $1,3 \mathrm{ab}$ & $1,8 \mathrm{ab}$ & $0,41 \mathrm{a}$ \\
\hline 9 & 29 ce & $4,2 \mathrm{bc}$ & $0,8 \mathrm{a}$ & $1,4 \mathrm{~cd}$ & $2,2 \mathrm{bc}$ & $0,57 \mathrm{~b}$ & $27 \mathrm{bc}$ & $4,5 \mathrm{ab}$ & $0,7 \mathrm{a}$ & $1,7 \mathrm{a}$ & $2,4 \mathrm{a}$ & $0,41 \mathrm{a}$ \\
\hline 10 & $26 \mathrm{e}$ & $4,3 \mathrm{bc}$ & $0,9 \mathrm{a}$ & $1,5 \mathrm{c}$ & $2,4 \mathrm{ac}$ & $0,59 \mathrm{~b}$ & $26 \mathrm{c}$ & $4,7 \mathrm{a}$ & $0,6 \mathrm{a}$ & $1,5 \mathrm{ab}$ & $2,1 \mathrm{ab}$ & $0,40 \mathrm{a}$ \\
\hline 11 & $28 \mathrm{de}$ & $4,3 \mathrm{bc}$ & $0,9 \mathrm{a}$ & $1,7 \mathrm{bc}$ & $2,6 \mathrm{ac}$ & $0,53 \mathrm{~b}$ & $26 \mathrm{bc}$ & $4,7 \mathrm{a}$ & $0,6 \mathrm{a}$ & $1,4 a b$ & $2,0 \mathrm{ab}$ & $0,43 \mathrm{a}$ \\
\hline Tr. & \multicolumn{6}{|c|}{ Peltophorum dubium } & \multicolumn{6}{|c|}{ Gallesia gorarema } \\
\hline 1 & $12 \mathrm{~d}$ & $3,4 \mathrm{~d}$ & $0,6 \mathrm{~b}$ & $1,1 \mathrm{e}$ & $1,6 \mathrm{~d}$ & $0,53 \mathrm{a}$ & $13 \mathrm{f}$ & $3,9 \mathrm{~d}$ & $0,4 \mathrm{c}$ & $0,5 \mathrm{~d}$ & $0,9 \mathrm{~d}$ & $0,73 \mathrm{a}$ \\
\hline 2 & $17 \mathrm{c}$ & $4,5 \mathrm{bc}$ & $0,7 \mathrm{ab}$ & $1,7 \mathrm{de}$ & $2,4 \mathrm{~cd}$ & 0,38 be & $19 \mathrm{e}$ & $5,0 \mathrm{bc}$ & $0,8 \mathrm{ab}$ & $1,2 \mathrm{c}$ & $2,0 \mathrm{c}$ & $0,69 \mathrm{ab}$ \\
\hline 3 & $21 \mathrm{ab}$ & $4,8 \mathrm{ac}$ & $1,0 \mathrm{a}$ & $2,8 \mathrm{ac}$ & $3,7 \mathrm{ab}$ & $0,35 \mathrm{ce}$ & $26 \mathrm{ab}$ & $5,8 \mathrm{ab}$ & $1,0 \mathrm{a}$ & $2,0 \mathrm{a}$ & $3,0 \mathrm{ab}$ & $0,49 \mathrm{~cd}$ \\
\hline 4 & $23 \mathrm{a}$ & $5,0 \mathrm{ab}$ & $0,8 \mathrm{ab}$ & $2,9 \mathrm{ab}$ & $3,7 \mathrm{ab}$ & $0,29 \mathrm{de}$ & $27 \mathrm{a}$ & $5,7 \mathrm{ab}$ & $1,0 \mathrm{a}$ & $2,0 \mathrm{a}$ & $3,1 \mathrm{a}$ & $0,49 \mathrm{~cd}$ \\
\hline 5 & $24 \mathrm{a}$ & $5,0 \mathrm{ab}$ & $0,7 \mathrm{ab}$ & $2,5 \mathrm{ad}$ & $3,2 \mathrm{ac}$ & $0,28 \mathrm{e}$ & $24 \mathrm{bc}$ & $4,8 \mathrm{c}$ & $0,6 \mathrm{bc}$ & $1,8 \mathrm{ab}$ & $2,4 \mathrm{ac}$ & $0,33 \mathrm{~d}$ \\
\hline 6 & $18 \mathrm{c}$ & $4,4 \mathrm{c}$ & $0,8 \mathrm{ab}$ & $1,7 \mathrm{ad}$ & $2,5 \mathrm{bd}$ & $0,45 \mathrm{ac}$ & $21 \mathrm{ce}$ & $5,2 \mathrm{ac}$ & $0,8 \mathrm{ab}$ & $1,1 \mathrm{c}$ & $1,9 \mathrm{c}$ & $0,68 \mathrm{ab}$ \\
\hline 7 & $17 \mathrm{c}$ & $4,3 \mathrm{c}$ & $0,9 a b$ & 1,9 be & $2,8 \mathrm{ad}$ & $0,47 \mathrm{ab}$ & $19 \mathrm{e}$ & $5,1 \mathrm{bc}$ & $0,8 \mathrm{ab}$ & $1,4 b c$ & $2,2 \mathrm{bc}$ & $0,61 \mathrm{ac}$ \\
\hline 8 & $19 \mathrm{bc}$ & $4,5 \mathrm{bc}$ & $0,7 \mathrm{ab}$ & $1,8 \mathrm{ce}$ & $2,6 \mathrm{bd}$ & $0,40 \mathrm{bd}$ & $21 \mathrm{de}$ & $5,1 \mathrm{bc}$ & $0,7 \mathrm{ac}$ & $1,3 \mathrm{c}$ & $2,0 \mathrm{c}$ & $0,55 \mathrm{bc}$ \\
\hline 9 & $21 \mathrm{ab}$ & $4,8 \mathrm{ac}$ & $0,7 \mathrm{ab}$ & $1,8 \mathrm{ce}$ & $2,5 \mathrm{bd}$ & 0,38 be & $21 \mathrm{de}$ & $5,2 \mathrm{ac}$ & $0,9 \mathrm{ab}$ & $1,4 \mathrm{bc}$ & $2,3 \mathrm{ac}$ & $0,64 \mathrm{ac}$ \\
\hline 10 & $23 \mathrm{a}$ & $5,2 \mathrm{a}$ & $1,0 \mathrm{a}$ & $2,4 \mathrm{ad}$ & $3,4 \mathrm{ac}$ & $0,42 \mathrm{bc}$ & $21 \mathrm{de}$ & $5,8 \mathrm{ab}$ & $0,9 \mathrm{ab}$ & $1,6 \mathrm{ac}$ & $2,5 \mathrm{ac}$ & $0,56 \mathrm{ac}$ \\
\hline 11 & $23 \mathrm{a}$ & $5,2 \mathrm{a}$ & $1,0 \mathrm{a}$ & $3,0 \mathrm{a}$ & $4,0 \mathrm{a}$ & 0,35 ce & $23 \mathrm{~cd}$ & $5,9 \mathrm{a}$ & $1,0 \mathrm{a}$ & $2,0 \mathrm{a}$ & $3,0 \mathrm{ab}$ & $0,53 \mathrm{bc}$ \\
\hline Tr. & \multicolumn{6}{|c|}{ Myroxylon peruiferum } & & & & & & \\
\hline 1 & $15 \mathrm{e}$ & $3,5 \mathrm{e}$ & $0,8 \mathrm{a}$ & $1,1 \mathrm{~d}$ & $1,8 \mathrm{~d}$ & $0,72 \mathrm{a}$ & & & & & & \\
\hline 2 & $19 \mathrm{~d}$ & $4,2 \mathrm{~d}$ & $1,0 \mathrm{a}$ & $2,0 \mathrm{ac}$ & $3,0 \mathrm{ac}$ & $0,48 \mathrm{~b}$ & & & & & & \\
\hline 3 & $22 \mathrm{bc}$ & $4,5 \mathrm{bc}$ & $1,0 \mathrm{a}$ & $2,7 \mathrm{a}$ & $3,6 \mathrm{a}$ & $0,36 \mathrm{~b}$ & & & & & & \\
\hline 4 & $25 \mathrm{ab}$ & $4,6 \mathrm{~b}$ & $0,9 \mathrm{a}$ & $2,5 \mathrm{a}$ & $3,4 \mathrm{ab}$ & $0,36 \mathrm{~b}$ & & & & & & \\
\hline 5 & $24 \mathrm{ab}$ & $4,5 \mathrm{bc}$ & $0,9 \mathrm{a}$ & $2,2 \mathrm{ac}$ & $3,1 \mathrm{ac}$ & $0,43 \mathrm{~b}$ & & & & & & \\
\hline 6 & $21 \mathrm{~cd}$ & $4,3 \mathrm{~cd}$ & $0,9 \mathrm{a}$ & $2,0 \mathrm{ab}$ & $3,0 \mathrm{ac}$ & $0,46 \mathrm{~b}$ & & & & & & \\
\hline 7 & $20 \mathrm{~d}$ & $4,2 \mathrm{~cd}$ & $0,7 \mathrm{a}$ & $1,5 \mathrm{~cd}$ & $2,3 \mathrm{~cd}$ & $0,48 \mathrm{~b}$ & & & & & & \\
\hline 8 & $19 \mathrm{~d}$ & $4,1 \mathrm{~d}$ & $0,8 \mathrm{a}$ & $1,7 \mathrm{bc}$ & $2,5 \mathrm{bd}$ & $0,48 \mathrm{~b}$ & & & & & & \\
\hline 9 & $23 \mathrm{bc}$ & $4,5 \mathrm{~b}$ & $1,0 \mathrm{a}$ & $2,3 a b$ & $3,3 \mathrm{ac}$ & $0,41 \mathrm{~b}$ & & & & & & \\
\hline 10 & $22 \mathrm{bc}$ & $4,6 \mathrm{~b}$ & $0,9 \mathrm{a}$ & $2,5 \mathrm{a}$ & $3,4 \mathrm{ab}$ & $0,36 \mathrm{~b}$ & & & & & & \\
\hline 11 & $26 \mathrm{a}$ & $4,9 \mathrm{a}$ & $1,0 \mathrm{a}$ & $2,6 \mathrm{a}$ & $3,6 \mathrm{a}$ & $0,39 \mathrm{~b}$ & & & & & & \\
\hline
\end{tabular}

Tratamentos: 1) sem adubação; 2) $1,6 \mathrm{~kg} / \mathrm{m}^{3}$ ALC; 3) $3,2 \mathrm{~kg} / \mathrm{m}^{3}$ ALC; 4) 4,8 kg/m $\mathrm{m}^{3}$ ALC; 5) convenc. CESP; 6) $1,6 \mathrm{~kg} / \mathrm{m}^{3}$ ALC + $0,75 \mathrm{~kg} / \mathrm{m}^{3} \mathrm{AP}$; 7) $\left.1,6 \mathrm{~kg} / \mathrm{m}^{3} \mathrm{ALC}+1,5 \mathrm{~kg} / \mathrm{m}^{3} \mathrm{AP} ; 8\right) 1,6 \mathrm{~kg} / \mathrm{m}^{3} \mathrm{ALC}+2,25 \mathrm{~kg} / \mathrm{m}^{3} \mathrm{AP} ;$ 9) $1,6 \mathrm{~kg} / \mathrm{m}^{3} \mathrm{ALC}+1,5 \mathrm{~kg} / \mathrm{m}^{3} \mathrm{AP}+0,5 \mathrm{~g} / 1 \mathrm{ACP}$; 10) $1,6 \mathrm{~kg} / \mathrm{m}^{3} \mathrm{ALC}+1,5 \mathrm{~kg} / \mathrm{m}^{3}$ AP $+1 \mathrm{~g} / \mathrm{l} \mathrm{ACP}$; e 11) $1,6 \mathrm{~kg} / \mathrm{m}^{3} \mathrm{ALC}+1,5 \mathrm{~kg} / \mathrm{m}^{3} \mathrm{AP}+2 \mathrm{~g} / 1 \mathrm{ACP}$. As letras diferentes nas colunas, dentro de cada espécie, diferem estatisticamente $(\mathrm{P} \leq 0,05)$ pelo teste de Tukey. 
capacidade fisiológica de crescimento da planta para as cinco espécies, refletindo também nas outras variáveis medidas (Quadro 5). A estabilização do crescimento de Guazuma ulmifolia e Croton floribundus, submetidos ao tratamento 4, aos 90 dias após a germinação (Figura 1), provavelmente foi devido ao limite imposto pelo volume do substrato ao sistema radicular das plantas.

O menor crescimento em altura das plantas dessas duas espécies no tratamento 5, comparado ao do tratamento 4 , pode ter sido motivado por um desequilíbrio nutricional em relação às necessidades das plantas. Uma das possibilidades poderia ser a maior lixiviação do $\mathrm{P}$ na forma de superfosfato simples (tratamento 5) do que $\mathrm{P}$ revestido por camada de resina (tratamento 4 ), especialmente se o pH do substrato for inferior a 6,0. Cole \& Dole (1997), em experimento de lixiviação de diferentes fontes de $\mathrm{P}$ em Tagetes erecta, acondicionados em potes de 1 litro (15 cm de diâmetro e $15 \mathrm{~cm}$ de altura) preenchidos com casca de pinus: turfa: areia na proporção volumétrica de 3:1:1, observaram após 50 dias do plantio que a quantidade de $\mathrm{P}$ lixiviado na forma de superfosfato simples foi três vezes superior àquela na forma de $\mathrm{P}$ revestido com resina. Por sua vez, Spinks \& Pritchett (1956) demonstraram que $P$ solúvel em água move-se prontamente através do perfil de um substrato orgânico se o pH está abaixo de 6,0, contudo em substratos com $\mathrm{pH}$ acima de 6,0, o movimento do $\mathrm{P}$ é restrito. A equipe deste estudo, em teste preliminar com mudas de Croton floribundus de 60 dias após a germinação (dados nãopublicados), usando o mesmo substrato, tipo de recipiente e adubação convencional (tratamento 5) do presente trabalho, observou que após a aplicação de adubação de cobertura com sulfato de amônio o pH determinado ficou abaixo de 6,0 .

A aplicação de $1,5 \mathrm{~kg} / \mathrm{m}^{3}$ de adubo em pó (AP) + $1,6 \mathrm{~kg} / \mathrm{m}^{3}$ de ALC (tratamento 7) resultou em um crescimento equivalente ao do tratamento 5 para o Croton floribundus (Figura 1), dando indicativos que a adubação

Quadro 6 - Concentração de nutrientes $(\mathrm{g} / \mathrm{kg})$ da parte aérea das mudas das cinco espécies Table 6 - Shoot nutrient concentration $(\mathrm{g} / \mathrm{kg}$ ) of the five species seedlings

\begin{tabular}{|c|c|c|c|c|c|c|c|c|c|c|c|c|}
\hline \multirow{2}{*}{ Tr. } & \multicolumn{6}{|c|}{ Guazuma ulmifolia } & \multicolumn{6}{|c|}{ Croton floribundus } \\
\hline & $\mathrm{N}$ & $P$ & $\mathrm{~K}$ & $\mathrm{Ca}$ & $\mathrm{Mg}$ & $\mathrm{S}$ & $\mathrm{N}$ & $\mathrm{P}$ & $\mathrm{K}$ & $\mathrm{Ca}$ & $\mathrm{Mg}$ & $S$ \\
\hline 1 & $8,6 \mathrm{ab}$ & $4,7 \mathrm{a}$ & $9,7 \mathrm{a}$ & $14,8 \mathrm{a}$ & $7,2 \mathrm{a}$ & $1,3 \mathrm{a}$ & $11,7 \mathrm{~b}$ & $3,1 \mathrm{a}$ & $9,2 \mathrm{a}$ & $12,5 \mathrm{ab}$ & $7,9 \mathrm{a}$ & $1,3 \mathrm{~b}$ \\
\hline 2 & $7,0 \mathrm{~b}$ & $3,2 \mathrm{~b}$ & $5,2 \mathrm{bc}$ & $11,7 \mathrm{~b}$ & $6,8 \mathrm{ab}$ & $1,4 \mathrm{a}$ & $10,0 \mathrm{~b}$ & $2,9 \mathrm{a}$ & $7,2 \mathrm{c}$ & $13,0 \mathrm{a}$ & $6,4 \mathrm{~b}$ & $1,3 \mathrm{~b}$ \\
\hline 3 & $7,5 \mathrm{ab}$ & $2,3 \mathrm{bc}$ & $5,9 \mathrm{~b}$ & $9,1 \mathrm{c}$ & $4,6 \mathrm{~cd}$ & $1,1 \mathrm{a}$ & $10,2 \mathrm{~b}$ & $1,8 \mathrm{~b}$ & $6,9 \mathrm{c}$ & $9,1 \mathrm{bc}$ & $3,7 \mathrm{~d}$ & $1,2 \mathrm{~b}$ \\
\hline 4 & $7,6 \mathrm{ab}$ & $2,0 \mathrm{c}$ & $4,9 \mathrm{c}$ & $6,8 \mathrm{~d}$ & 3,2 de & $1,0 \mathrm{a}$ & $10,3 \mathrm{~b}$ & $2,0 \mathrm{~b}$ & $7,5 \mathrm{c}$ & $8,6 \mathrm{c}$ & $2,9 \mathrm{~d}$ & $1,1 \mathrm{~b}$ \\
\hline 5 & $9,4 \mathrm{a}$ & $2,3 \mathrm{bc}$ & $8,9 \mathrm{a}$ & $7,4 \mathrm{~cd}$ & $3,0 \mathrm{e}$ & $1,1 \mathrm{a}$ & $16,8 \mathrm{a}$ & $2,0 \mathrm{~b}$ & $9,4 \mathrm{a}$ & $10,1 \mathrm{ac}$ & $3,7 \mathrm{~d}$ & $2,4 \mathrm{a}$ \\
\hline 7 & $7,3 \mathrm{~b}$ & $2,3 \mathrm{bc}$ & $5,5 \mathrm{bc}$ & $9,0 \mathrm{c}$ & $5,5 \mathrm{bc}$ & $1,4 \mathrm{a}$ & $10,2 \mathrm{~b}$ & $2,0 \mathrm{~b}$ & $7,4 \mathrm{c}$ & $12,0 \mathrm{ac}$ & $5,3 \mathrm{c}$ & $1,3 \mathrm{~b}$ \\
\hline 11 & $7,8 \mathrm{ab}$ & $1,9 \mathrm{c}$ & $5,4 \mathrm{bc}$ & $7,4 \mathrm{~cd}$ & 4,4 ce & $1,0 \mathrm{a}$ & $12,0 \mathrm{~b}$ & $2,0 \mathrm{~b}$ & $8,4 \mathrm{~b}$ & $11,0 \mathrm{ac}$ & $5,0 \mathrm{c}$ & $1,4 \mathrm{~b}$ \\
\hline Tr. & \multicolumn{6}{|c|}{ Peltophorum dubium } & \multicolumn{6}{|c|}{ Gallesia integrifolia } \\
\hline 1 & $10,5 \mathrm{ac}$ & $2,4 \mathrm{a}$ & $5,5 \mathrm{bc}$ & $8,7 \mathrm{a}$ & $2,5 \mathrm{a}$ & $1,4 \mathrm{~b}$ & $15,1 \mathrm{bc}$ & $4,0 \mathrm{bc}$ & $8,6 \mathrm{e}$ & $14,5 \mathrm{a}$ & $9,0 \mathrm{a}$ & $2,7 \mathrm{~b}$ \\
\hline 2 & $8,0 \mathrm{c}$ & $2,0 \mathrm{a}$ & $4,9 \mathrm{c}$ & $7,1 \mathrm{ab}$ & $1,8 \mathrm{~b}$ & $1,3 \mathrm{~b}$ & $12,8 \mathrm{c}$ & $5,5 \mathrm{a}$ & $11,1 \mathrm{c}$ & $12,7 \mathrm{ab}$ & $9,2 \mathrm{a}$ & $2,5 \mathrm{~b}$ \\
\hline 3 & $10,9 \mathrm{ac}$ & $2,3 \mathrm{a}$ & $6,1 \mathrm{ab}$ & $7,6 \mathrm{ab}$ & $2,1 \mathrm{ab}$ & $1,7 \mathrm{ab}$ & $12,7 \mathrm{c}$ & $4,6 \mathrm{ab}$ & $9,1 \mathrm{de}$ & $12,0 \mathrm{ab}$ & $7,7 \mathrm{ab}$ & $2,4 \mathrm{bc}$ \\
\hline 4 & $11,4 \mathrm{ab}$ & $2,4 \mathrm{a}$ & $6,0 \mathrm{ab}$ & $7,1 \mathrm{ab}$ & $2,0 \mathrm{ab}$ & $1,6 \mathrm{ab}$ & $13,7 \mathrm{c}$ & $3,5 \mathrm{bc}$ & $8,6 \mathrm{e}$ & $11,7 \mathrm{~b}$ & $6,6 \mathrm{~b}$ & $1,6 \mathrm{~d}$ \\
\hline 5 & $13,2 \mathrm{a}$ & $2,3 \mathrm{a}$ & $6,8 \mathrm{a}$ & $6,9 a b$ & $1,7 \mathrm{~b}$ & $2,0 \mathrm{a}$ & $24,1 \mathrm{a}$ & $3,1 \mathrm{c}$ & $17,3 \mathrm{a}$ & $5,6 \mathrm{c}$ & $2,9 \mathrm{c}$ & $3,8 \mathrm{a}$ \\
\hline 7 & $8,4 \mathrm{bc}$ & $2,0 \mathrm{a}$ & $4,8 \mathrm{c}$ & $6,5 \mathrm{~b}$ & $1,6 \mathrm{~b}$ & $1,3 \mathrm{~b}$ & $14,0 \mathrm{bc}$ & $3,3 \mathrm{bc}$ & $9,9 \mathrm{~d}$ & $11,8 \mathrm{ab}$ & $8,4 \mathrm{ab}$ & $1,8 \mathrm{~cd}$ \\
\hline 11 & $11,9 \mathrm{a}$ & $2,0 \mathrm{a}$ & $6,2 \mathrm{ab}$ & $6,6 \mathrm{~b}$ & $2,0 \mathrm{ab}$ & $1,2 \mathrm{~b}$ & $16,5 \mathrm{~b}$ & $4,5 \mathrm{ab}$ & $12,3 \mathrm{~b}$ & $13,0 \mathrm{ab}$ & $9,5 \mathrm{a}$ & $2,7 \mathrm{~b}$ \\
\hline Tr. & \multicolumn{6}{|c|}{ Myroxylon peruiferum } & & & & & & \\
\hline 1 & $10,0 \mathrm{~d}$ & $2,2 \mathrm{a}$ & $7,5 \mathrm{~b}$ & $10,2 \mathrm{a}$ & $1,9 \mathrm{ab}$ & $1,1 \mathrm{~cd}$ & & & & & & \\
\hline 2 & $10,7 \mathrm{~d}$ & $2,0 \mathrm{a}$ & $7,1 \mathrm{~b}$ & $9,9 \mathrm{ab}$ & $2,2 \mathrm{a}$ & $1,2 \mathrm{c}$ & & & & & & \\
\hline 3 & $12,2 \mathrm{c}$ & $1,8 \mathrm{a}$ & $7,1 \mathrm{~b}$ & $8,6 \mathrm{ac}$ & $1,9 \mathrm{ab}$ & $1,2 \mathrm{c}$ & & & & & & \\
\hline 4 & $10,4 \mathrm{~d}$ & $1,8 \mathrm{a}$ & $6,6 \mathrm{bc}$ & $7,9 \mathrm{bc}$ & $1,7 \mathrm{ab}$ & $1,4 \mathrm{~b}$ & & & & & & \\
\hline 5 & $17,2 \mathrm{a}$ & $1,8 \mathrm{a}$ & $11,4 \mathrm{a}$ & $7,4 \mathrm{c}$ & $1,6 \mathrm{~b}$ & $1,6 \mathrm{a}$ & & & & & & \\
\hline 7 & $10,4 \mathrm{~d}$ & $1,9 \mathrm{a}$ & $6,0 \mathrm{c}$ & $9,5 \mathrm{ab}$ & $2,2 \mathrm{a}$ & $1,0 \mathrm{~d}$ & & & & & & \\
\hline 11 & $15,4 \mathrm{~b}$ & $1,9 \mathrm{a}$ & $10,9 \mathrm{a}$ & $9,1 \mathrm{ac}$ & $2,2 \mathrm{a}$ & $1,1 \mathrm{~cd}$ & & & & & & \\
\hline
\end{tabular}

As letras diferentes nas colunas, dentro de cada espécie, diferem estatisticamente $(\mathrm{P} \leq 0,05)$ pelo teste de Tukey. 
Quadro 7 - Conteúdo de nutrientes (mg) da parte aérea das mudas das cinco espécies Table 7 - Shoot nutrient content ( $\mathrm{mg}$ ) of the five species seedlings

\begin{tabular}{|c|c|c|c|c|c|c|c|c|c|c|c|c|}
\hline \multirow{2}{*}{ Tr. } & \multicolumn{6}{|c|}{ Guazuma ulmifolia } & \multicolumn{6}{|c|}{ Croton floribundus } \\
\hline & $\mathrm{N}$ & $\mathrm{P}$ & $\mathrm{K}$ & $\mathrm{Ca}$ & $\mathrm{Mg}$ & $\mathrm{S}$ & $\mathrm{N}$ & $\mathrm{P}$ & $\mathrm{K}$ & $\mathrm{Ca}$ & $\mathrm{Mg}$ & $\mathrm{S}$ \\
\hline 1 & $22 \mathrm{~d}$ & $12 a b c$ & $25 \mathrm{~cd}$ & $38 \mathrm{bc}$ & $18 \mathrm{~b}$ & $3 \mathrm{c}$ & $25 \mathrm{~b}$ & $6 \mathrm{~b}$ & $19 \mathrm{~b}$ & $26 \mathrm{~b}$ & $17 \mathrm{a}$ & $3 \mathrm{~b}$ \\
\hline 2 & $32 \mathrm{~cd}$ & $14 \mathrm{a}$ & $24 \mathrm{~cd}$ & $53 \mathrm{ab}$ & $31 \mathrm{a}$ & $6 \mathrm{ab}$ & $42 \mathrm{~b}$ & $12 \mathrm{a}$ & $30 \mathrm{ab}$ & $55 \mathrm{ab}$ & $27 \mathrm{a}$ & $5 \mathrm{~b}$ \\
\hline 3 & $50 \mathrm{ab}$ & $15 \mathrm{a}$ & $40 \mathrm{~b}$ & $61 \mathrm{a}$ & $31 \mathrm{a}$ & $7 \mathrm{a}$ & $51 \mathrm{~b}$ & $9 \mathrm{ab}$ & $34 \mathrm{ab}$ & $45 \mathrm{ab}$ & $18 \mathrm{a}$ & $6 \mathrm{~b}$ \\
\hline 4 & $53 \mathrm{ab}$ & $14 \mathrm{a}$ & $34 \mathrm{bc}$ & $48 \mathrm{abc}$ & $22 \mathrm{~b}$ & $7 \mathrm{ab}$ & $52 \mathrm{ab}$ & $10 \mathrm{ab}$ & $38 \mathrm{ab}$ & $44 \mathrm{ab}$ & $15 \mathrm{a}$ & $6 \mathrm{~b}$ \\
\hline 5 & $63 \mathrm{a}$ & $15 \mathrm{a}$ & $59 a$ & $49 a b c$ & $20 \mathrm{~b}$ & $7 a$ & $80 \mathrm{a}$ & $9 a b$ & $45 \mathrm{a}$ & $48 \mathrm{ab}$ & $18 \mathrm{a}$ & $11 \mathrm{a}$ \\
\hline 7 & $27 \mathrm{~cd}$ & $8 \mathrm{c}$ & $20 \mathrm{~d}$ & $33 \mathrm{c}$ & $20 \mathrm{~b}$ & $5 \mathrm{bc}$ & $51 \mathrm{~b}$ & $10 \mathrm{ab}$ & $37 \mathrm{ab}$ & $60 \mathrm{a}$ & $26 \mathrm{a}$ & $6 \mathrm{~b}$ \\
\hline 11 & $40 \mathrm{bc}$ & $10 \mathrm{bc}$ & $28 \mathrm{bcd}$ & $38 \mathrm{bc}$ & $22 \mathrm{~b}$ & $5 \mathrm{bc}$ & $50 \mathrm{~b}$ & $8 \mathrm{ab}$ & $35 \mathrm{ab}$ & $46 \mathrm{ab}$ & $21 \mathrm{a}$ & $6 \mathrm{~b}$ \\
\hline Tr. & \multicolumn{6}{|c|}{ Peltophorum dubium } & \multicolumn{6}{|c|}{ Gallesia integrifolia } \\
\hline 1 & $33 \mathrm{~b}$ & $7 c$ & $17 \mathrm{c}$ & $27 \mathrm{~b}$ & $8 \mathrm{c}$ & $4 d$ & $23 \mathrm{e}$ & $6 \mathrm{~d}$ & $13 \mathrm{~d}$ & $22 \mathrm{~d}$ & $13 \mathrm{~d}$ & $4 \mathrm{e}$ \\
\hline 2 & $41 \mathrm{~b}$ & $10 \mathrm{bc}$ & $25 \mathrm{bc}$ & $37 \mathrm{~b}$ & $9 \mathrm{c}$ & $7 \mathrm{~cd}$ & $45 \mathrm{de}$ & $19 \mathrm{abc}$ & $39 \mathrm{c}$ & $44 \mathrm{~cd}$ & $32 \mathrm{bcd}$ & $9 \mathrm{de}$ \\
\hline 3 & $90 \mathrm{a}$ & $19 \mathrm{a}$ & $50 \mathrm{a}$ & $63 \mathrm{a}$ & $17 \mathrm{a}$ & $14 \mathrm{a}$ & $78 \mathrm{bc}$ & $28 \mathrm{a}$ & $55 \mathrm{bc}$ & $73 \mathrm{ab}$ & $47 \mathrm{ab}$ & $15 \mathrm{bc}$ \\
\hline 4 & $98 \mathrm{a}$ & $21 \mathrm{a}$ & $52 \mathrm{a}$ & $61 \mathrm{a}$ & $17 \mathrm{a}$ & $14 \mathrm{a}$ & $84 \mathrm{bc}$ & $21 \mathrm{abc}$ & $53 \mathrm{bc}$ & $72 \mathrm{ab}$ & $40 \mathrm{ab}$ & $10 \mathrm{~cd}$ \\
\hline 5 & $99 \mathrm{a}$ & $17 \mathrm{a}$ & $51 \mathrm{a}$ & $52 \mathrm{a}$ & $13 \mathrm{~b}$ & $15 \mathrm{a}$ & $133 \mathrm{a}$ & $17 \mathrm{bc}$ & $95 \mathrm{a}$ & $31 \mathrm{~cd}$ & $16 \mathrm{~cd}$ & $21 \mathrm{a}$ \\
\hline 7 & $49 \mathrm{~b}$ & $12 \mathrm{~b}$ & $28 \mathrm{~b}$ & $38 \mathrm{~b}$ & $9 \mathrm{c}$ & $8 c$ & $57 \mathrm{~cd}$ & $13 \mathrm{~cd}$ & $40 c$ & $48 \mathrm{bc}$ & $34 \mathrm{bc}$ & $7 \mathrm{de}$ \\
\hline 11 & $106 \mathrm{a}$ & $18 \mathrm{a}$ & $55 \mathrm{a}$ & $59 \mathrm{a}$ & $18 \mathrm{a}$ & $11 \mathrm{~b}$ & $97 \mathrm{~b}$ & $26 \mathrm{ab}$ & $72 a b$ & $76 \mathrm{a}$ & $56 \mathrm{a}$ & $16 \mathrm{ab}$ \\
\hline Tr. & \multicolumn{6}{|c|}{ Myroxylon peruiferum } & & & & & & \\
\hline 1 & $32 \mathrm{~d}$ & $7 \mathrm{c}$ & $24 \mathrm{~d}$ & $33 \mathrm{~d}$ & $6 c$ & $3 c$ & & & & & & \\
\hline 2 & $66 \mathrm{bcd}$ & $12 \mathrm{ab}$ & $44 \mathrm{bcd}$ & $61 \mathrm{bc}$ & $13 \mathrm{ab}$ & $7 a b$ & & & & & & \\
\hline 3 & $97 \mathrm{ab}$ & $14 \mathrm{a}$ & $57 \mathrm{ab}$ & $69 \mathrm{a}$ & $15 \mathrm{a}$ & $10 \mathrm{a}$ & & & & & & \\
\hline 4 & $79 \mathrm{abc}$ & $14 \mathrm{a}$ & $50 \mathrm{bc}$ & $60 \mathrm{abc}$ & $13 \mathrm{ab}$ & $11 \mathrm{a}$ & & & & & & \\
\hline 5 & $112 \mathrm{a}$ & $12 \mathrm{ab}$ & $74 \mathrm{a}$ & $48 \mathrm{bcd}$ & $10 \mathrm{~b}$ & $10 \mathrm{a}$ & & & & & & \\
\hline 7 & $48 \mathrm{~cd}$ & $9 \mathrm{bc}$ & $28 \mathrm{~cd}$ & $44 \mathrm{~cd}$ & $10 \mathrm{bc}$ & $5 \mathrm{bc}$ & & & & & & \\
\hline 11 & $111 \mathrm{a}$ & $14 \mathrm{a}$ & $79 \mathrm{a}$ & $66 \mathrm{ab}$ & $16 \mathrm{a}$ & $8 \mathrm{ab}$ & & & & & & \\
\hline
\end{tabular}

As letras diferentes nas colunas, dentro de cada espécie, diferem estatisticamente $(\mathrm{P} \leq 0,05)$ pelo teste de Tukey.

de cobertura com sulfato de amônio no tratamento 5 resultou em consumo de luxo, como atesta a concentração de N e S no Quadro 6. Por sua vez, o crescimento em altura de Guazuma ulmifolia no tratamento 5 foi maior que no tratamento 7 , provavelmente pelo efeito de uma maior utilização do $\mathrm{N}$ nas vias metabólicas, onde a pequena diferença entre as concentrações de $\mathrm{N}$ nesses dois tratamentos parece suportar essa hipótese (Quadro 6).

Por outro lado, quando se compara o tratamento 11 $\left[1,5 \mathrm{~kg} / \mathrm{m}^{3} \mathrm{AP}+1,6 \mathrm{~kg} / \mathrm{m}^{3} \quad \mathrm{ALC}+2,0 \mathrm{~g} / \mathrm{l}\right.$ de ACP (adubação de cobertura periódica)] com o tratamento 7 para o Peltophorum dubium, a Gallesia integrifolia e o Myroxylon peruiferum (Figura 1), nota-se que os nutrientes aplicados no substrato, no tratamento 7, provavelmente esgotaram-se aos 60 dias (época em que se iniciou a estabilização do crescimento em altura) e que, em contrapartida, a adubação de cobertura do tratamento 11 nas mudas resultou em crescimento adicional em altura.

Assim, Hicklenton \& Cairns (1996) desenvolveram um estudo sobre métodos de irrigação em viveiro para Cotoneaster dammeri e Forsythia ovata x Forsythia europaea, onde usaram substrato constituído por casca de pinus, turfa e areia, recipientes de 3,8 litros e adubo de liberação controlada. Verificaram que o método que apresentou menos lixiviação, sem causar estresse hídrico para as mudas, resultou em plantas com maior biomassa seca da parte aérea para as duas espécies, 16 semanas após o transplante para o recipiente. Contudo, nem sempre menor lixiviação é sinônimo de maior crescimento da planta, ou seja, se for mantida uma alta condutividade elétrica (CE) no substrato, o crescimento pode ser prejudicado. Huett (1997) realizou um experimento de lixiviação em plantas de quatro semanas de crescimento, onde usaram substrato composto por $50 \%$ de casca de

R. Árvore, Viçosa-MG, v.27, n.6, p.779-789, 2003 
pinus compostada, $30 \%$ de pó de serra compostada, $20 \%$ de areia grossa de rio (v/v), recipiente plástico de $335 \mathrm{ml}$ e adubo de liberação controlada. Observou-se nos substratos onde não houve lixiviação que as plantas tiveram menor biomassa seca da parte aérea do que nos substratos cujo volume lixiviado a cada dois dias era de $200 \mathrm{ml}$, onde as condutividades elétricas ao final de quatro semanas foram 2,1 e $0,88 \mathrm{mS} / \mathrm{cm}$, respectivamente.

Observa-se no Quadro 6 que a concentração de $\mathrm{Mg}$ na parte aérea das mudas no tratamento 5 (convencional), onde foi aplicado sulfato de amônio a cada 14 dias, foi uma das mais baixas em todas as espécies, especialmente para a Gallesia integrifolia. Este fato pode estar relacionado com a competição do $\mathrm{Mg}$ com o $\mathrm{NH}_{4}$. A inibição da absorção de $\mathrm{Mg}$ por $\mathrm{NH}_{4}$, quando comparado com $\mathrm{NO}_{3}$, pode ser um mero reflexo da competição por cargas negativas dentro de células individuais, ou na planta inteira (Marschner, 1995). Contudo, a concentração de Mg na parte aérea das mudas de todas as espécies no tratamento $4\left(4,8 \mathrm{~kg} / \mathrm{m}^{3}\right.$ de ALC) não diferiu da do tratamento 5. A concentração da solução do substrato imediatamente antes da repicagem (Quadro 4) no tratamento 4 apresentou 0,6 e $1,7 \mathrm{mg} / \mathrm{l}$ de nitrogênio amoniacal e nítrico, respectivamente, e nessas condições pode ter havido uma absorção preferencial do $\mathrm{NH}_{4}$, que pode ter inibido a absorção de Mg. Quando ambas as fontes de $\mathrm{N}$ estão presentes na solução, a planta preferencialmente absorve $\mathrm{NH}_{4}$, se a razão entre $\mathrm{NH}_{4}$ e $\mathrm{NO}_{3}$ for acima de 0,1 (Kafkafi, 1990).

Por sua vez, também pode ter havido competição do K e Ca contra o Mg. Duboc et al. (1996) e Braga et al. (1995), em experimentos de omissão de nutrientes em mudas de espécies arbóreas, observaram, quando se omitiu Ca ou K para a Hymenaea courbaril var. stilbocarpa (jatobá) no primeiro ensaio e quando se omitiu Ca para a Tibouchina granulosa (quaresmeira), a Aspidosperma polyneuron (peroba-rosa) e o Platycyamus regnellii (paupereira) no segundo ensaio, que houve aumento na concentração de $\mathrm{Mg}$ na parte aérea das mudas.

A maior concentração de $\mathrm{Mg}$ na parte aérea (Quadro 6) de Guazuma ulmifolia e Croton floribundus submetidas ao tratamento 1 (testemunha), em relação aos outros tratamentos, pode ter refletido a maior concentração de $\mathrm{P}$. A absorção de $\mathrm{H}_{2} \mathrm{PO}_{4}$ é máxima na presença do $\mathrm{Mg}$ : esse papel de 'carregador de fósforo' possivelmente pode ser explicado pela sua participação na ativação de ATPases da membrana implicadas na absorção iônica (Malavolta et al., 1997). Por sua vez, a maior concentração de $\mathrm{Mg}$ na parte aérea dessas espécies na

R. Árvore, Viçosa-MG, v.27, n.6, p.779-789, 2003 testemunha concorda com resultados de Hunt \& Walker (1991), onde folhas de Pinus jeffrey e Pinus monophylla no tratamento sem adubação apresentaram maior concentração de $\mathrm{Mg}$ e Ca, quando comparados com tratamentos onde foram aplicados adubos de liberação controlada.

A maior concentração de S (Quadro 6) na parte aérea de Croton floribundus, Gallesia integrifolia e Myroxylon peruiferum no tratamento 5, em comparação com outros tratamentos, pode ter sido estimulada pela provável baixa concentração de nitrato durante a fase experimental (Quadro 4). A absorção dos ânions $\mathrm{Cl}, \mathrm{SO}_{4}$ e $\mathrm{H}_{2} \mathrm{PO}_{4}$ pode ser estimulada quando a absorção de $\mathrm{NO}_{3}$ é fortemente deprimida (Mengel \& Kirkby, 1987).

\section{CONCLUSÕES}

Os tratamentos que utilizaram adubo de liberação controlada (19-06-10, N- $\mathrm{P}_{2} \mathrm{O}_{5}-\mathrm{K}_{2} \mathrm{O}$ ) nas doses de $3,2 \mathrm{~kg} / \mathrm{m}^{3}$ (T3) e $4,8 \mathrm{~kg} / \mathrm{m}^{3}$ (T4) resultaram em mudas de boa qualidade para todas as espécies. Para a Guazuma ulmifolia o tratamento convencional da CESP (adubação de base mais adubação de cobertura com sulfato de amônio e cloreto de potássio, T5) também poderia ser recomendado. Quanto às outras quatro espécies (Croton floribundus, Peltophorum dubium, Gallesia integrifolia e Myroxylon peruiferum), a utilização da combinação de adubo de liberação controlada $\left(1,6 \mathrm{~kg} / \mathrm{m}^{3}\right.$ de $\left.19-06-10\right)$ mais adubo em pó $\left(1,5 \mathrm{~kg} / \mathrm{m}^{3}\right.$ de $\left.14-16-18\right)$ mais adubação de cobertura periódica ( $2 \mathrm{~g} / \mathrm{l}$ de 19-06-20), tratamento 11, proporcionou a obtenção de mudas de crescimento similar às dos tratamentos de maiores doses de adubo de liberação controlada (T3 e T4).

\section{REFERÊNCIAS BIBLIOGRÁFICAS}

AMERICAN PUBLIC HEALTH ASSOCIATION - APHA. Standard methods for the examination of water and waste water. 14.ed. Washington: APHA, 1976. 1193 p.

BRAGA, F. A. et al. Exigências nutricionais de quatro espécies florestais. Revista Árvore, v. 19, n. 1, p. 18-31, 1995.

BUDOWSKI, G. Distribution of tropical American rain forest species in the light of successional processes. Turrialba, v. 15, p. 40-42, 1965.

COLE, J. C.; DOLE, J. M. Temperature and phosphorus source affect phosphorus retention by a pine bark-based container medium. HortScience, v. 32, n. 2, p. 236-240, 1997. 
COX, D. A. Reducing nitrogen leaching losses from containerised nursery plants: the effectiveness of controlled release fertilisers. Journal of Plant Nutrition, v. 16, p. 533-545, 1993.

DUBOC, E. et al. Nutrição do jatobá (Hymenaea courbaril L. var. stilbocarpa (Hayne) Lee et Lang.). Cerne, v. 2, n. 1, p. 138-152, 1996.

HAMILTON, D. F.; JOHNSON, C. R. Effects of organic matter and controlled-release fertilizer on nutrient retention during intermittent-mist propagation. Scientia

Horticulturae, v. 8, n. 2, p. 155-162, 1978.

HICKLENTON, P. R.; CAIRNS, K. G. Plant water relations and mineral nutrition of containerized nursery plants in relation to irrigation method. Canadian Journal Plant Science, v. 76, n. 1, p. 155-160, 1996.

HUETT, D. O. Fertiliser use efficiency by containerised nursery plants 1 . Plant growth and nutrient uptake.

Australian Journal of Agriculture Research, v. 48, n. 2, p. 251-258, 1998.

HUETT, D. O.; MORRIS, S. C. Fertiliser use efficiency by containerised nursery plants. 3 . Effect of heavy leaching and damaged fertiliser prills on plant growth, nutrient uptake, and nutrient loss. Australian Journal Agricultural Research, v. 50, n. 2, p. 217-222, 1999.

HUNT, C. D.; WALKER, R. F. Growth and mineral nutrition of containerized jeffrey pine and single leaf pinyon as affected by controlled-release fertilization. Tree Planters' Notes, v. 42, n. 1, p. 27-33, 1991.
KAFKAFI, U. Root temperature, concentration and the ratio $\mathrm{NO}_{3}{ }^{-} / \mathrm{NH}_{4}{ }^{+}$effect on plant development. Journal of Plant Nutrition, v. 13, n. 10, p. 1291-1306, 1990.

MALAVOLTA, E.; VITTI, G. C.; OLIVEIRA, S. A. Avaliação do estado nutricional das plantas: princípios e aplicações. 2.ed. Piracicaba: POTAFOS, 1997. 319 p.

MARSCHNER, H. Mineral nutrition of higher plants. 2.ed. London: Academic Press, 1995. 889 p.

MENGEL, K.; KIRKBY, E. A. Principles of plant nutrition. 4.ed. Bern: International Potash Institute, 1987. $687 \mathrm{p}$.

RODELlA, A. A.; ALCARDE, J. C. Requisitos de qualidade física e química de fertilizantes minerais. In: GONÇALVES, J. L. M.; BENEDETTI, V. Nutrição e fertilização florestal. Piracicaba: IPEF, 2000. p. 59-78.

SAS INSTITUTE. SAS procedures guide, version 6. 4.ed. Cary, North Carolina, 1990.

SPINKS, D. O.; PRITCHETT, W. L. The downward movement of phosphorus in potting soils as measured by $\mathrm{P}^{32}$. Proceedings Florest State Horticultural Society, v. 69, p. 385-388, 1956.

STEEL, R. D.; TORRIE, J. H. Principles and procedures of statistics: a biometrical approach. 2.ed. New York: McGraw Hill, 1980. 633 p. 